\title{
Les Nouveaux modes d'engagement par la photographie en Grande-Bretagne
}

New Modes of Commitment through Photography in Britain

\section{Karine Chambefort-Kay}

\section{(2) OpenEdition}

\section{Journals}

Édition électronique

URL : http://journals.openedition.org/rfcb/1487

DOI : $10.4000 /$ rfcb. 1487

ISSN : 2429-4373

Éditeur

CRECIB - Centre de recherche et d'études en civilisation britannique

Référence électronique

Karine Chambefort-Kay, "Les Nouveaux modes d'engagement par la photographie en GrandeBretagne », Revue Française de Civilisation Britannique [En ligne], XXII-3 | 2017, mis en ligne le 05 juillet 2017, consulté le 02 mai 2019. URL : http://journals.openedition.org/rfcb/1487 ; DOI : 10.4000/ rfcb. 1487

Ce document a été généré automatiquement le 2 mai 2019.

\section{(i) $\$$}

Revue française de civilisation britannique est mis à disposition selon les termes de la licence Creative Commons Attribution - Pas d'Utilisation Commerciale - Pas de Modification 4.0 International. 


\title{
Les Nouveaux modes d'engagement par la photographie en Grande- Bretagne
}

New Modes of Commitment through Photography in Britain

\author{
Karine Chambefort-Kay
}

\section{Introduction}

1 La notion de "photographie engagée" s'est évaporée du champ des études photographiques à la fin des années 1980 et avec elle, la figure héroïque du reporter engagé1. Dans les vingt dernières années, elle semble être devenue une idée démodée, voire quelque peu suspecte. Les lectures poststructuralistes des images ont en effet pointé les rapports de domination que pouvait supposer l'acte photographique, qui assujettit son objet au regard du photographe, pour le soumettre à celui des spectateurs, tandis que les réflexions sur la réception ont montré les limites de l'empathie chez ces derniers et évoqué, comme Susan Sontag, la possibilité d'une compassion fatigue ${ }^{2}$.

2 Cela ne signifie pas pour autant que le principe de l'engagement ait été évacué des pratiques photographiques contemporaines. L'engagement, en photographie comme dans tous les arts, est fondé sur l'idée qu'une image a un pouvoir de transformation de la société, qu'elle peut avoir une fonction, voire une responsabilité politique et sociale. Dans la Grande-Bretagne contemporaine, nombre d'œuvres, de projets et de pratiques mettent en œuvre un tel engagement par l'image photographique, parfois en dehors des circuits strictement artistiques ou journalistiques. Elles peuvent être le fait d'individus militants mais, de plus en plus souvent, ce sont des œuvres collectives, qui engagent non plus un, mais des photographes selon divers modes de participation. Nous souhaitons interroger ce qui nous semble être une résurgence des usages engagés de la photographie dans la période contemporaine et en particulier, leur dimension participative. À partir de trois études de cas - le projet New Londoners, de l'association Photovoice, la campagne I am an Immigrant de Mouvement Against Xenophobia et The Election Project de Simon Roberts - 
nous proposons de dégager une première typologie des modes d'engagement participatifs dans la photographie contemporaine britannique, et de discuter en quoi ils pourraient constituer des voies inédites.

3 Dans cette optique, nous commencerons par revenir sur la trajectoire de la notion de photographie engagée dans l'histoire de la photographie en Grande-Bretagne. Il s'agira notamment de discuter son apparent déclin dans les années 1990 pour mieux identifier ce qui, dans les pratiques photographiques des deux décennies suivantes, constituerait un renouveau ou une réinvention de l'engagement par la photographie. De manière transversale, nous serons amenés à situer ces pratiques dans leur contexte politique, social et culturel, mais aussi dans celui des évolutions techniques, en particulier l'émergence de nouveaux modes de partage et de communication sur les plateformes de de type Flickr et les réseaux sociaux. La dimension participative commune à la plupart des projets et les usages croissants des images comme vecteur même de l'expression en ligne nous invitent à réévaluer la relation entre images et engagement au XxI siècle.

\section{Trajectoire de la notion de photographie engagée en Grande-Bretagne}

4 La notion de "photographie engagée " fut d'abord employée en référence aux photojournalistes qui, parfois au péril de leur vie, rapportent de pays en guerre ou en situation de détresse humanitaire, des images fortes destinées à témoigner et à faire réagir le lectorat de la presse généraliste et de magazines illustrés, public généralement lui-même à l'abri de ces difficultés ${ }^{3}$. De ce point de vue, l'âge d'or de la photographie engagée pourrait se situer entre les décennies 1930 et 1970 où, de guerre civile espagnole en conflit mondial et en guerre froide, les crises se sont succédé et déroulées en images dans Picture Post, LIFE et autres. De grands reporters ont marqué leur temps, comme les gallois Philip Jones Griffiths ${ }^{4}$ et Don Mc Cullin ${ }^{5}$, actifs pendant la guerre du Vietnam mais aussi, par exemple, pendant la grande famine du Biafra à la fin des années 1960 pour le second ${ }^{6}$.

Néanmoins, la notion de photographie engagée renvoie aussi très souvent à la tradition documentaire lorsque celle-ci se saisit de thématiques sociales pour susciter une prise de conscience chez son public et militer pour des réformes sociales. Dans ce cas, on pourrait remonter aux origines de la photographie et aux premiers travaux documentaires, comme ceux de Thomas Annan ou John Thompson pour la Grande-Bretagne. On pense aussi à l'entreprise du nom de Mass Observation lancée en 1937, dont faisait partie le photographe Humphrey Spender, et qui se donna pour but de montrer « de manière simple à tous les observateurs, que leur environnement peut être compris, et donc constamment transformé. Quelles que soient les méthodes politiques mises en oeuvre pour effectuer cette transformation, une bonne connaissance de ce qui doit être transformé est indispensable ${ }^{7}$. ». Dans l'après-guerre, la photographie dite humaniste procède d'une même intention. Les images documentaires accompagnent souvent des articles engagés en faveur de réformes sociales comme celui intitulé " The Forgotten Gorbals », publié par Picture Post en janvier 1948 avec treize photos de Bill Brandt et Bert Hardy prises dans le quartier populaire des Gorbals à Glasgow. À Londres, Roger Mayne réalise un travail similaire à Southam Street ${ }^{8}$. L'intention de la photographie engagée, est, à ce moment-là, en adéquation avec les aspirations sociales de l'après-guerre. 
6 À partir des années 1970, la prétention à l'objectivité cette photographie dite documentaire est remise en question par un groupe informel d'artistes et de théoriciens dont le Canadien Allan Sekula est la figure de proue. Ils affirment que les pratiques documentaires de l'époque moderne, comme celles de Jacob Riis ou de la Farm Security Administration aux États-Unis, "s'abritaient derrière une idéologie bidon de la neutralité ${ }^{\text {. } ~ » ~ E n ~}$ Grande-Bretagne, John Tagg formule la critique la plus radicale: ce que dénonce cet auteur très influent, c'est le paternalisme à l'œuvre dans la photographie documentaire engagée, qui donne bonne conscience à son public de classe moyenne en plaidant pour des réformes à la marge mais qui ne remettent pas en cause radicalement l'ordre social. Ce paternalisme réside notamment dans le regard distancié et la neutralité dont se targuent les photographes documentaires, regard désormais critiqué pour son caractère condescendant, qui assujettit les personnes photographiées alors que l'intention initiale est bien de plaider leur cause. Le réalisme et la capacité des images documentaires à œuvrer à de véritables transformations sociales sont désormais sujets à caution.

7 La conséquence de cette critique radicale est de rendre suspect le travail sur un groupe social, c'est-à-dire un travail depuis l'extérieur. Il faudrait au contraire travailler avec, travailler de l'intérieur, c'est à dire s'engager plus littéralement, ou mieux, engager les sujets des images à devenir acteurs de leur représentation pour s'émanciper du regard du photographe. Le mode participatif apparaît alors comme une réponse naturelle à cette nouvelle conception. De multiples expériences en la matière se développent en photographie au cours des années 1970, sous la bannière de "community arts», par exemple dans les quartiers de Tower Hamlets ou de Handsworth, comme l'a montré Mathilde Bertrand ${ }^{10}$. L'expérience, quoique foisonnante, reste brève, faute de moyens pendant la période thatchérienne. Le climat politique reste néanmoins un fort facteur d'engagement chez de nombreux photographes qui renouvellent le documentaire social, notamment en faisant usage pour la première fois de la couleur, comme Paul Graham ou Paul Reas.

8 Le cas de Martin Parr, dans la même mouvance à l'origine, est emblématique de la crise que connaît ensuite cette photographie engagée, après une décennie de questionnement. La nomination de ce photographe britannique à l'agence Magnum en 1994 et la violente polémique interne qu'elle suscite sont un tournant symbolique. En effet, certains, dont Phillip Jones Griffith, ancien président de la coopérative, ou Henri Cartier-Bresson, membre fondateur, voient dans le style de Martin Parr un dévoiement du style de reportage que développe Magnum depuis sa création par Robert Capa. Parr et sa posture souvent sarcastique sont en rupture avec la figure héroïque du reporter engagé qui a fait les riches heures de l'agence.

Ce que cristallise cet épisode, c'est surtout une perte de repères irréversible quant à la forme documentaire elle-même et à ses capacités d'engagement. D'une part, elle n'est plus nécessairement synonyme de militantisme; et d'autre part, il apparaît à la même période qu'elle n'est plus le vecteur privilégié de l'engagement en photographie. En effet, elle a cédé du terrain à d'autres explorations formelles du côté du mode allégorique, psychanalytique ou de la fiction, dont la puissance politique n'est pas moindre. En Grande-Bretagne, les autoportraits intimes et néanmoins très engagés réalisés par Jo Spence à la fin des années 1980 sont un exemple de cette évolution.

10 L'exemple de Jo Spence témoigne aussi d'une hybridation des thématiques de l'engagement en photographie. Les motivations sont désormais moins strictement sociales mais se font l'écho des questionnements et des luttes féministes, ou encore du 
mouvement LGBT. Enfin, nous avons montré ailleurs qu'à partir des années 1990, l'engagement en photographie concerne plus volontiers les questions de représentation des minorités dans une Grande-Bretagne qui se décrit désormais comme une société multiculturelle ${ }^{11}$. Une nouvelle génération de photographes documente la vie des jeunes britanniques d'origine afro-caribéenne, indienne ou pakistanaise (Faisal Abdu Allah, Jagtar Semplay, Rehan Jamil, Suki Dhanda) et pose, de façon plus ou moins radicale, la question de l'intégration des minorités dites de la deuxième et de la troisième génération en Grande-Bretagne. D'autres s'intéressent à l'accueil des immigrés (Dinu Li, Anthony Lam) et d'autres encore, comme Yinka Shonibare, utilisent la photographie pour mettre en question l'invisibilité des minorités afro-caribéennes dans la culture visuelle en Grande-Bretagne et invoquer une réécriture l'histoire de l'art britannique. Sur le plan thématique, la notion d'engagement en photographie s'est donc considérablement élargie à la fin du vingtième siècle en Grande-Bretagne.

\section{Résurgence des collectifs de photographes : pour quel engagement?}

11 Depuis les années 2000, on observe à nouveau la formation de multiples collectifs de photographes en Grande-Bretagne. Il serait impossible d'en dresser ici la liste exhaustive. Citons les principaux: Documenting Britain, Kennardphillipps, Document Scotland, A Fine Beginning, the Photographers Collective, sans compter les collectifs internationaux auxquels participent des Britanniques comme In-Public, crée en 2000 dédié à la photographie de rue.

On peut être tenté de faire le parallèle avec la période des années 1970 où les photographes s'organisèrent en formations semblables. C'est ce qu'a semblé suggérer l'association Photoworks, organisatrice de la Biennale de Photographie de Brighton, en choisissant pour l'édition de 2014 le thème : Communities, Collectives \& Collaboration ${ }^{12}$. Parmi les expositions proposées, on trouvait en effet une rétrospective sur l'agence Magnum destinée à « réinterpréter la façon dont les inclinations sociales, culturelles et politiques ont forgé cette archive de renommée mondiale ", mais aussi une exposition tirée du fonds d'archive de la Mass Observation, et enfin, une autre encore sur le groupe Co-Optic actif dans les années $1970^{13}$. Concernant la période contemporaine, étaient présentés les travaux de cinq collectifs contemporains ${ }^{14}$ dont la caractéristique commune est de travailler de manière collaborative : il s'agissait de « souligner l'impact de cette façon de travailler, du point de vue national et international. Ce qui est présenté ici, c'est le processus dynamique et démocratique consistant à naviguer et à négocier pour arriver à une expression cohérente à travers la notion de collectif15." Enfin, nombre d'ateliers étaient organisés à l'intention des habitants de Brighton et Hove, comme celui intitulé «A view to the Past » animé par Lydia Goldblatt, qui proposait aux participants une activité sur les stéréotypes en photographie. De tels ateliers sont la marque de fabrique de la biennale, à l'initiative de l'association Photoworks, qui selon sa propre définition «se consacre à favoriser la participation en photographie, le média le plus démocratique de la culture visuelle contemporaine ${ }^{16}$ . » La programmation 2014 offrait donc un remarquable panorama sur quantité d'actions et d'œuvres autour des notions de collectif, de collaboration et de participation.

Le risque, néanmoins, qui n'a pas été complètement évité par Photoworks nous semble-til, était de renforcer l'amalgame entre ces notions déjà courant chez les critiques, voire 
chez les artistes eux-mêmes. Cela pose deux problèmes si l'on en revient à la question de l'engagement: d'une part, l'amalgame entre collaboratif et participatif ne permet pas d'interroger réellement les ressorts propres à chaque mode de travail, et donc, leur pouvoir en termes d'engagement, de mobilisation, ou d'action politique et socioculturelle. D'autre part, le rapprochement avec des collectifs antérieurs très politisés contribue sans doute à surestimer la capacité et la volonté d'engagement de ces formations contemporaines. Celles-là procèdent certes à une mutualisation des moyens utiles à la diffusion de leurs travaux, notamment via les réseaux sociaux, et parfois à l'organisation d'expositionss de groupe (voir par exemple l'exposition annuelle du Photographerscollective ou les travaux du collectif Photodebut, actif de 2002 à $2009^{17}$ ) ; mais peu conçoivent des objectifs communs au-delà de la visibilité accrue des projets individuels de leurs membres. Le collectif Documenting Britain, par exemple, se contente de demander à chacun de ses membres une contribution mensuelle sur son blog à propos de ses activités personnelles en cours. Enfin, certains collectifs sont formés par des étudiants en photographie dans le cadre de travaux communs et sont assez éphémères comme The Forgotten Collective, créé à Glasgow en 2013, mais inactif depuis août 2015 mais sont un moyen pour les jeunes photographes de se confronter aux enjeux de l'exposition de groupe et d'être solidaires au moment du passage crucial vers la pratique professionnelle.

Rares sont donc les collectifs qui affichent une vision commune et s'engagent pour la promouvoir dans des manifestes explicites. Notons l'exception de kennardphillips, association de Peter Kennard et de Cat Phillips, dont l'objectif est on ne peut plus clair :

a collaboration working since 2002 to produce art in response to the invasion of Iraq. It has evolved to confront power and war across the globe. The work is made for the street, the gallery, the web, newspapers \& magazines, and to lead workshops that develop peoples' skills and help them express their thoughts on what's happening in the world through visual means. The work is made as a critical tool that connects to international movements for social and political change. We don't see the work as separate to social and political movements that are confronting established political and economic systems. We see it as part of those movements, the visual arm of protest. We want it to be used by people as a part of their own activism, not just as pictures on the wall to contemplate [...].

15 On peut aussi signaler la formation de collectifs sur des lignes nationales, comme Document Scotland et son équivalent gallois A Fine Beginning. Le premier, formé par quatre photographes (Sophie Gerrard, Jeremy Sutton-Hibbert, Colin McPherson et Stephen McLaren) se donne pour mission d'explorer l'identité écossaise par la photographie comme le stipule sa page de présentation :

Nous sommes passionnés de photographie documentaire et nous sommes engagés dans la représentation de l'Écosse. [...] Aujourd'hui, en quelque sorte, c'est l'appel de la Calédonie. [...] L'Écosse se trouve à un moment décisif de son histoire. Les événements des années à venir seront déterminants pour nos relations avec nos voisins et le reste du monde. Document Scotland est convaincu que la photographie peut et devrait jouer un rôle central pour conserver une trace de cette période. Notre ambition est de produire des travaux et de contribuer à un récit qui constituera plus tard un composant indispensable de l'histoire et du dialogue dans notre nation. Nous espérons laisser un document visuel, un témoignage sur la période extraordinaire que nous vivons ${ }^{18}$.

La participation du collectif à l'exposition Document Scotland: The Ties That Bind qui s'est tenue à la National Portrait Gallery of Scotland à Edimbourg en 2015-2016 témoigne de 
son engagement dans la production d'une vision internaliste de l'Écosse et du soutien institutionnel qu'il a reçu en Écosse.

C'est un exemple de la mobilisation récente de la photographie sur les thématiques identitaires et nationales plutôt que sociale. Néanmoins, quoi que ce collectif jouisse d'une certaine visibilité en vertu du contexte politique et constitutionnel, un tel engagement et une vision commune aussi explicite restent une exception dans le paysage photographique. Globalement, nous concluons que la résurgence récente des collectifs photographiques que nous venons de présenter ne saurait être interprétée par un calque sur les périodes antérieures. Si la multiplication des collectifs aujourd'hui est incontestable, on ne peut y voir pour autant une re-politisation massive du champ de la photographie depuis les années 2010.

\section{Le mode participatif}

18 Nous avons souligné que le mode collaboratif et le mode participatif méritaient d'être distingués et mis en regard. Tournons-nous donc maintenant vers les pratiques photographiques participatives développées en Grande-Bretagne à partir des années 2000. Comme les collectifs de photographes, elles connaissent un renouveau, à la faveur des nouvelles possibilités offertes par la photographie numérique et les outils de partage d'images en ligne. Offrent-elles plus de matière à l'engagement par la photographie ?

19 Notons tout d'abord que ce renouveau ne se restreint pas à la photographie seule mais concerne l'ensemble des arts contemporains. L'historien de l'art Nicolas Bourriaud a décrit cette tendance dès 1998 et proposé le concept « d'esthétique relationnelle » pour rendre compte de ces nouvelles pratiques artistiques, une «théorie esthétique consistant à juger les cuvres d'art en fonction des relations interhumaines qu'elles figurent, produisent ou suscitent.» Nicolas Bourriaud observe en effet au tournant du millénaire une reconfiguration du terrain de l'art contemporain, dans un «ensemble de pratiques artistiques qui prennent pour point de départ théorique et pratique l'ensemble des relations humaines et leur contexte social, plutôt qu'un espace autonome et privatif. ${ }^{19}$ ". Certaines pratiques participatives favorisent donc une perméabilité plus grande entre sphère artistique et sphère politique et sociale. Qu'en est-il des pratiques photographiques, sachant que le medium photographique se situe déjà, par nature, à l'intersection de ces différentes sphères? La photographie parvient-elle à s'engager davantage sur le terrain des relations humaines et sociales en optant pour le mode participatif?

Nous proposons d'étudier maintenant trois cas de pratiques photographiques participatives situées justement en dehors du champ strictement artistique pour mettre en lumière les modalités de l'engagement qui s'y joue, notamment en termes d'émancipation, d'identification collective et de mobilisation politique.

\section{Émancipation par l'image : le cas de Photovoice}

21 La collaboration entre sujet photographié et photographe est au cœur du projet New Londoners mené par l'organisation caritative Photovoice. Cette ONG créée en 2003 par Anna Blackman et Tiffany Fairey a pour mission de "donner une voix ", un moyen d'expression et de représentation aux personnes marginalisées dans le monde, par le biais 
de la photographie. Exclus, victimes de conflits, réfugiés, personnes handicapées participent à des formations et ateliers photographiques qui leur permettent de raconter leur histoire en images, d'exprimer leur point de vue, rarement représenté.

Depuis sa création, Photovoice mène des actions auprès des réfugiés mineurs de Londres, en partenariat avec une autre organisation, DOST, basée au Trinity Community Centre dans le quartier d'East Ham, qui accompagne quelque 500 réfugiés chaque année avec des cours d'anglais et autres dispositifs de soutien. Plusieurs programmes de Photovoice se sont succédé à partir de 2002 : Transparency (2002-2003) a impliqué treize mineurs dans un projet de représentation photographique de Londres. Le programme a reçu une certaine visibilité par un reportage sur $\mathrm{BBC}$ Londres et une exposition à la National Portrait Gallery. Un autre programme du nom de Moving Lives, en partenariat cette fois avec le British Refugee Council, a renouvelé l'expérience en 2006 : 36 jeunes réfugiés ont produit des vidéos en faisant équipe avec des jeunes des quartiers de Newham, Redbridge et Barking.

En 2007, le programme connaît un nouveau développement avec un système de tutorat par des photographes professionnels ${ }^{20}$. Quinze d'entre eux parrainent individuellement de jeunes réfugiés âgés de treize à vingt-trois ans, originaires d'une dizaine de pays, qui vivent à Londres, pour certains, depuis six mois seulement, pour d'autres, depuis six ans. Pendant une période d'environ quatre mois, les photographes s'engagent à former ces jeunes au maniement d'un appareil photo numérique avant de les accompagner dans la réalisation d'images sur leur expérience de la vie à Londres. Ce dernier projet donne lieu à la publication d'un livre intitulé New Londoners, Reflections on Home ${ }^{21}$.

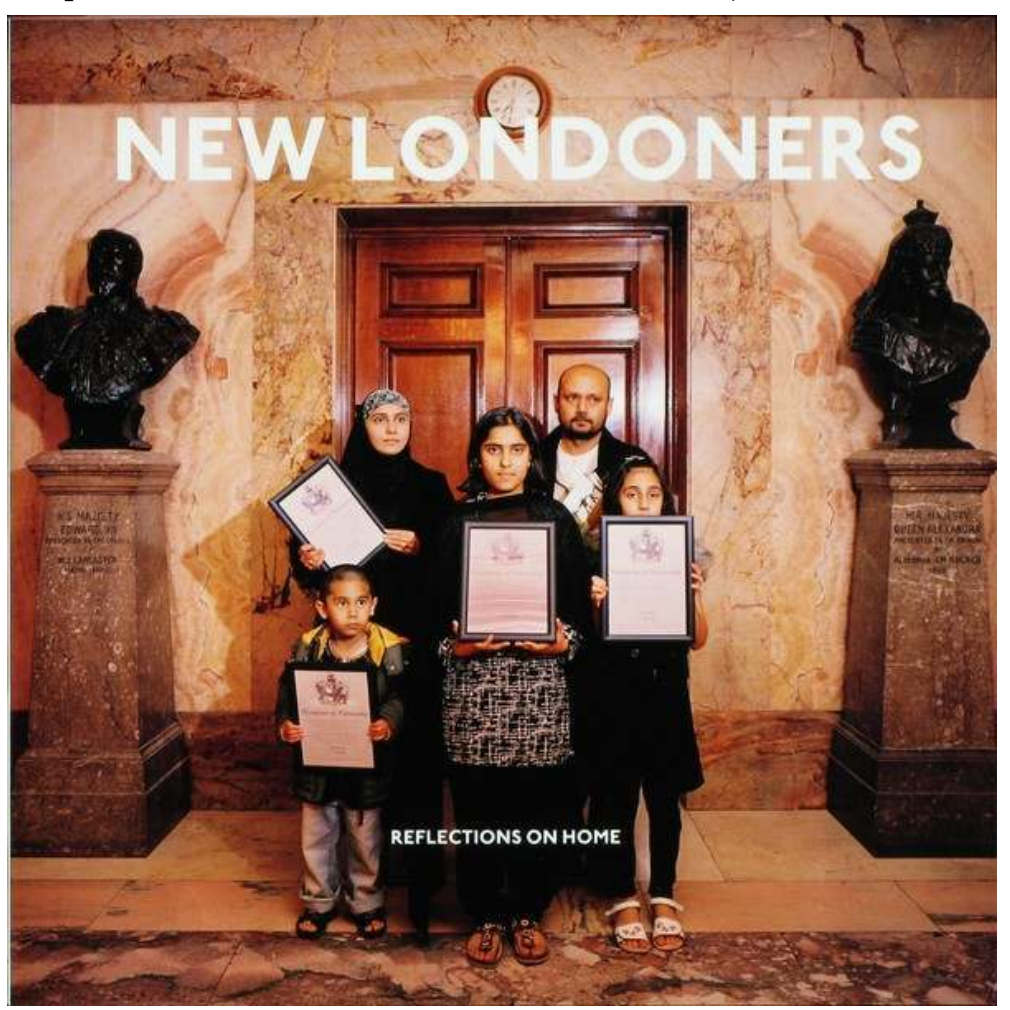

Couverture du livre New Londoners : Reflections on Home (2009),

Photo de Tatiana Correia, série New Citizens.

Pour les plus jeunes réfugiés et les plus récemment arrivés, cette forme de tutorat individuel par un adulte est une aide concrète à l'adaptation à un nouvel environnement. 
Cela ne remplace évidemment pas l'assistance matérielle, sanitaire, juridique et éducative apportée par les autres organisations auxquelles est associée Photovoice. Cependant, le simple fait pour les jeunes réfugiés de se rendre à des rendez-vous réguliers avec des photographes, de se déplacer dans Londres avec eux ou de visiter des expositions, par exemple, est un moyen de se créer des repères spatiaux et temporels. Le fait d'observer et de photographier des lieux est une façon de se les approprier. La réalisation d'autoportraits est aussi un moyen de s'inscrire littéralement dans le paysage pour ces jeunes qui se sont déracinés en quittant leur pays d'origine et leur famille. En outre, les photos sont par nature des enregistrements, des souvenirs de moments particuliers. Elles permettent aux réfugiés de se créer une histoire et des repères temporels dans leur nouvelle vie. "Le fait de prendre des photos est une façon d'établir qu'une nouvelle vie prend forme, en se confrontant aux ombres inquiétantes et en célébrant ce qui est beau. Ces images sont des souvenirs - et quand vous avez des souvenirs d'un endroit, vous commencez à avoir des racines 22 ", résume l'écrivain Hari Kunzru dans son introduction au livre.

Outre les aspects pratiques, le bénéfice humain de l'action conduite par Photovoice est donc essentiel. Il réside en particulier dans la possibilité qu'offre l'image numérique aux réfugiés qui maîtrisent peu l'anglais de communiquer de manière simple et de rompre leur isolement. L'apprentissage des techniques de la photographie aux côtés d'un professionnel est aussi très valorisant. La photographie est donc utilisée ici comme un outil d'intégration sur des plans très concrets, mais aussi comme un outil de reconquête de soi. En réalisant leurs propres images, les jeunes réfugiés ont la main sur leur représentation, ils en sont les agents. Hari Kunzru souligne que tout acte photographique requiert un certain nombre de choix (de sujet, de point de vue, de cadrage) et que, pour des jeunes qui n'ont eu que très peu de choix dans leur vie, se voir confier une telle responsabilité, même si elle peut paraitre futile, est précisément un acte d'empowerment ou d'émancipation. C'est un moyen de reprendre confiance en soi, en son pouvoir d'initiative, pour des jeunes dont le destin est encore suspendu à des décisions administratives de droit de séjour ou d'exclusion. Le jeune Irakien Chalak Abdulrahman exprime précisément cela dans le texte qui accompagne un paysage urbain rendu flou par la pluie qui ruisselle sur la vitre à travers laquelle il prend la photo : «Peut-être que je serai heureux ici. Je n'ai pas choisi d'être ici, et je n'ai pas le choix s'ils décident de me renvoyer. Je devrais être heureux mais je ne le suis pas. Je ne peux pas encore commencer ma vie. Tant que je n'ai pas mon permis de séjour ${ }^{23}$."

L'étude du projet New Londoners en tant qu'outil d'émancipation et d'intégration ne doit cependant pas conduire à une focalisation exclusive sur les processus à l'œuvre dans la création photographique et sur leurs effets possibles sur l'intégration des individus dans la société d'accueil. On pourrait d'ailleurs estimer que décrire a priori les bénéfices que tirent les réfugiés de leur participation au projet relève d'une forme de condescendance du même type que celle que l'on cherche à éviter par ce genre de projet participatif. Les effets produits par ces images sont donc à rechercher aussi du côté de ceux qui les reçoivent.

Dans son énumération des objectifs du livre New Londoners, une des deux coordinatrices du programme, Liz Orton, cite en premier lieu le fait de favoriser l'intégration des jeunes réfugiés et de mieux faire comprendre leur situation au public. Il s'agit de sensibiliser le monde politique et de calmer les débats sur les demandeurs d'asile, très vifs au milieu des années 2000 et attisés par une presse sensationnaliste : «Notre ambition est de contribuer à 
déplacer le débat sur les demandeurs d'asile pour sortir de la peur, de l'hostilité et de la différence et aller vers des points communs et une connaissance mutuell ${ }^{24}$. »

Ainsi, dès la deuxième page, Bajram Spahia, jeune réfugié résidant à Londres depuis 2001, confie :

Mes premières impressions de Londres étaient étranges. J'étais à East Ham, et ce n'était pas comme le Londres que j'avais vu à la télévision. De voir des coutumes et des modes de vie différents était une expérience nouvelle pour moi. Au bout d'un moment, j'ai appris à connaitre ces cultures et à les respecter. Une fois qu'on surmonte le côté étrange de la différence, on comprend que beaucoup de choses sont pareilles ${ }^{25}$.

On perçoit ici très clairement l'intérêt d'avoir le point de vue des réfugiés. Le spectateur comprend que ceux qui incarnent l'altérité pour la société d'accueil sont eux-mêmes aux prises avec l'altérité. Il se rend compte, aussi, que certains de ces jeunes sont les premiers surpris par la diversité qu'ils rencontrent à Londres. Ils n'ont jamais fait l'expérience d'un tel cosmopolitisme dans leur pays d'origine. Ils contribuent donc à la diversité de Londres, ils transforment la ville mais sont aussi transformés par elle, d'autant plus qu'ils sont encore de jeunes adultes. Voici ce qu'en dit l'Irakien Chalak Abdulrahman : «J'aime Londres parce qu'il y a toutes sortes de gens du monde entier qui vivent ensemble. Tous les gens sont égaux mais différents. Dans mon pays, tout le monde est pareil, nous sommes tous musulmans, mais nous nous battons. J'aime ma ville d'origine mais je veux vivre à Londres et être égal aux autres, quand j'aurai un permis de séjour. »

En somme, comme le soulignent Tiffany Fairey et Liz Orton dans leur avant-propos au livre, ces photographies "évoquent aussi une transition - d'un endroit à un autre, de la dépendance à l'indépendance, et d'un mode de vie à un autre ${ }^{26}$. Pour les réfugiés, les images et les textes produits sont des outils de transition et d'émancipation. Pour le public, elles sont des objets précieux pour la compréhension de telles transitions. Elles sont un moyen de s'émanciper des représentations dominantes et des attitudes de méfiance envers les réfugiés.

31 Toutefois, il faut souligner que de tels objets ne tombent pas dans les catégories esthétiques ou artistiques conventionnelles et trouvent difficilement des canaux de diffusion, au-delà d'un milieu déjà convaincu. Le deuxième cas que nous allons étudier maintenant, sur une thématique et des objectifs assez proches, exploite aussi le mode participatif mais cherche d'autres voies pour toucher un public plus grand.

\section{La campagne I AM AN IMMIGRANT : identification et mobilisation collective}

En mai 2015, la campagne I AM AN IMMIGRANT a produit et affiché dans l'espace public (400 stations de métro de Londres et 550 gares des grandes villes) une série de quinze posters en forme de fiches d'identité d'immigrés vivant en Grande-Bretagne: chaque affiche est constituée d'un portrait photographique réalisé par le photographe de Vogue Philip Volkers, des informations " Nom », « Pays d'origine » et " Métier », accompagnées d'une phrase présentant la contribution de cette personne à la communauté nationale.

Comme pour New Londoners, c'est l'objectif de lutter contre l'invisibilité, contre les stéréotypes et d'offrir une voix aux immigrés qui a présidé à l'organisation de cette campagne par le mouvement contre la xénophobie (MAX) soutenu par le Joint Council for the Welfare of Immigrants (JCWI) et par le Trades Union Congress (TUC) : « Notre campagne vise 
à interroger les stéréotypes négatifs, à mettre en avant les immigrés, et à leur fournir une plateforme pour partager leur histoire ${ }^{27}$. » Dans le contexte des élections générales de 2015, la question de l'immigration est un enjeu central avec le succès croissant du parti UKIP et l'intention de David Cameron de remettre en question la libre circulation des personnes dans l'Union Européenne.

La dimension participative de ce projet réside dans deux aspects : d'une part, dans la collecte de fonds organisée pour le financer via la plateforme de crowdfunding et d'autre part, dans la collecte de portraits réalisée via un site dédiée ${ }^{28}$. En effet, pour ajouter aux quinze posters officiels de la campagne, le public a été invité à créer ses propres fiches individuelles et à alimenter une banque d'images avec ses propres portraits. Accompagnées du slogan "I am an immigrant », les portraits ainsi déposés dans la base deviennent des autoportraits, même s'ils n'en sont pas toujours techniquement. Les sujets des affiches, les immigrés, sont auteurs de leur description et choisissent l'image qu'ils offrent d'eux-mêmes. De ce point de vue, les images et leur usage remplissent ici une fonction émancipatrice car elles mettent en œuvre des représentations directes, sans intermédiaire.

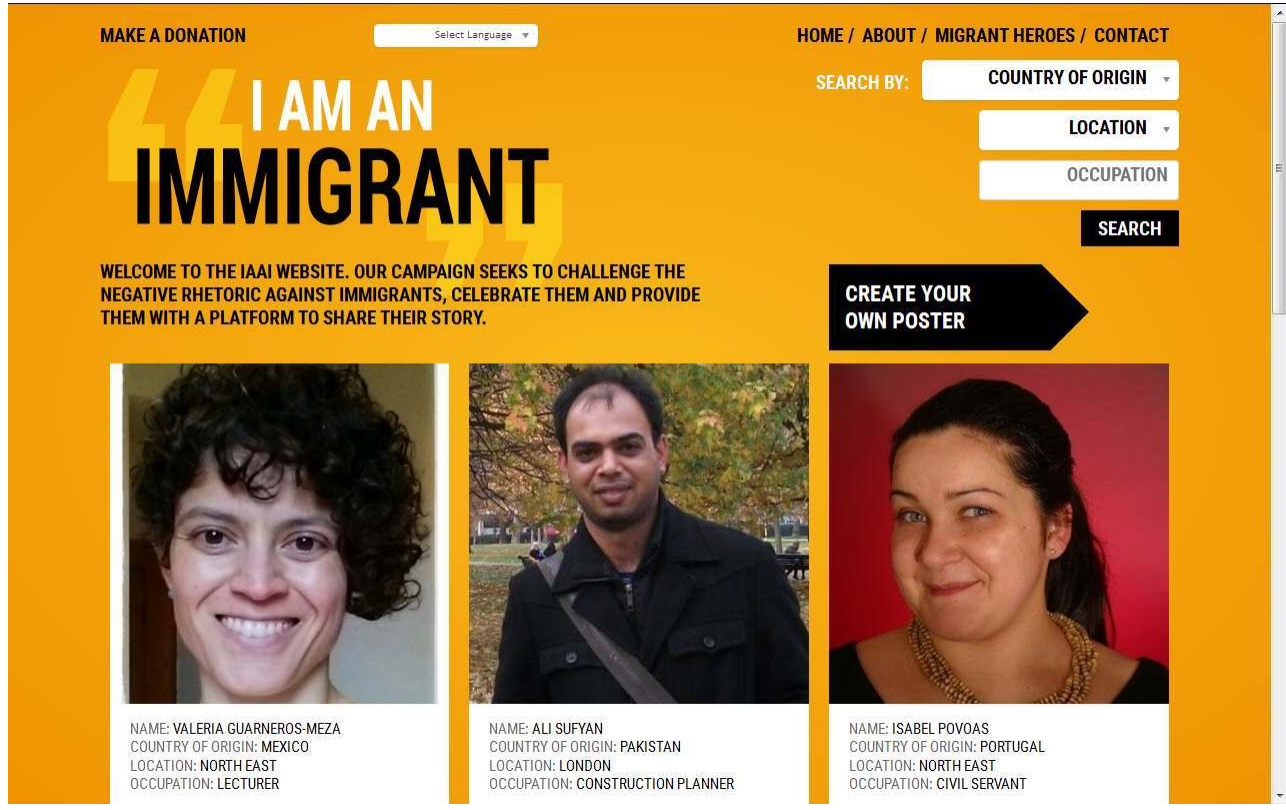

Capture d'écran de la page d'accueil du site de la campagne I AM AN IMMIGRANT réalisée en juin 2015.

Enfin, sur le site dédié à la campagne, on peut aussi observer la disposition des images, et la proximité qu'elle entretient avec les pages de réseaux sociaux comme LinkedIn ou encore avec des sites de rencontre, par un couplage entre photo et fiche synthétique. Du point de vue du spectateur, cette interface contribue à figurer ces fiches disparates comme un réseau personnel, fait d'amis et de contacts divers. En mimant les réseaux professionnels et amicaux, le site I AM AN IMMIGRANT souligne l'insertion des immigrés dans le tissu social et économique, comme veut le rappeler aussi le TUC qui soutient la campagne.

En somme, chaque portrait photographique est une forme d'auto-inscription, d'autodéclaration par l'image, renforcé par le slogan «I am an immigrant ». Outre les quelques informations fournies, ce sont les images qui sont les vecteurs de l'expression ici. Le mode participatif ainsi mis en œuvre consiste donc en une multitude de mobilisations 
individuelles, agrégées techniquement par le site mais aussi symboliquement par le spectateur, qui conçoit une trame virtuelle. L'engagement de chacun dans la campagne produit donc bien in fine une identification collective et une mobilisation, autour d'un statut, celui d'immigré, jusqu'ici peu mobilisateur en tant que tel en Grande-Bretagne.

Le dernier projet participatif que nous proposons d'étudier maintenant utilise des outils similaires de partage d'images, dans un contexte différent.

\section{The Election Project de Simon Roberts : photojournalisme citoyen et nouvelle sphère publique}

Pour les élections générales de 2010, le parlement britannique a décidé de mandater un photographe pour produire une œuvre en lien avec ce scrutin. Le Committee for the Works of Art de la Chambre des communes pratique cette forme de mécénat depuis 2001, sur une idée du ministre de la Culture Tony Banks. Avant chaque scrutin général, le comité lance un appel à projet et désigne un artiste officiel. Celui-ci peut donc suivre la campagne et avoir un accès privilégié aux différents candidats. Il a ensuite toute liberté pour produire une œuvre en lien avec les élections. Simon Roberts, qui vient de publier We English, est choisi parmi une liste de douze photographes soumise au comité de sélection par des commissaires et conservateurs en photographie. Son projet retient l'attention de la commission car il propose de suivre la campagne pendant vingt-cinq jours et de garder une image par jour, en se démarquant du suivi par les médias nationaux qui ont tendance à se focaliser sur les trois partis principaux et leurs dirigeants. Simon Roberts propose au contraire de s'intéresser aux petits partis, aux aspects triviaux de la campagne et aux stratégies déployées par les candidats pour aller à la rencontre des électeurs. Il intitule son oeuvre The Election Project.

$39 \mathrm{Au}$ fur et à mesure qu'il se déplace au Royaume-Uni, Simon Roberts invite les habitants des circonscriptions qu'il visite à prendre eux aussi des photos et à les partager. Ils peuvent déposer leurs images directement dans une galerie virtuelle sur son site ou les envoyer par mail et par SMS s'ils ont pris la photo avec leur téléphone portable. Toutes les images sont affichées, quelle que soit leur qualité. La visée n'est pas tant esthétique que politique. Le public est invité à produire son propre témoignage de ce moment de la vie démocratique du Royaume-Uni. Pour lancer son appel à participation en direction du public, Simon Roberts utilise la presse et les radios locales qui annoncent son passage dans les différentes circonscriptions et présentent son projet.

Le public est donc invité à pratiquer une sorte de journalisme "do-it-yourself», un journalisme autoproduit, ou un " journalisme citoyen » selon le terme de Simon Roberts, afin de rendre compte d'aspects peu connus de la campagne. Le mode participatif permet de rassembler une multiplicité de points de vue sur les élections dans une période où les grands médias, pris dans la concurrence de l'information en temps réel, ont tendance à reprendre les mêmes images en boucle et à se focaliser sur quelques événements spectaculaires, comme le « Duffy Gate » qui entache la campagne de Gordon Brown.

41 C'est donc confier à des amateurs la tâche de représenter la campagne. Ils n'ont pas les mêmes contraintes que les photojournalistes professionnels, qui sont envoyés en mission par les journaux avec un cahier des charges précis. Ils sont potentiellement plus libres et plus spontanés dans le choix des sujets et des moments photographiés. Les photographes amateurs locaux ont aussi une connaissance personnelle des lieux ou des personnes 
photographiées. Enfin, par le biais de la base participative, leurs images sont accessibles de façon directe, sans intermédiaire et sans processus d'édition. En somme, résume Simon Roberts,

le recours à des photographies prises par le public, téléchargées depuis des appareils numériques ou transmises par les téléphones portables, créera une vision photographique alternative en plus de la [sienne], produite avec le moyen plus traditionnel d'une chambre photographique, et cela ajoutera une dimension collaborative et démocratique à l'ensemble de l'œuvre. Ces images contribueront aussi à fournir un antidote aux photographies davantage mises en scène qui nous parviennent de plus en plus souvent des campagnes électorales ${ }^{29}$.

La plateforme nommée "The Public Gallery » au sein de The Election Project recueille ainsi 1696 photos dans le temps relativement court que dure la campagne officielle. Hormis quelques hors-sujets comme le cas d'une personne qui s'est obstinée à partager ses photos de vacances en Inde ou les abus d'un militant utilisant le projet comme un support de campagne, Simon Roberts relève que les contributeurs ont joué le jeu et qu'il a pu conserver toutes les images déposées dans la base. Le processus participatif ainsi mis en place reproduit d'une certaine façon le processus démocratique, puisqu'il accorde une légitimité à chaque participant. Comme l'a souligné Hugo Swire, président du Committee on Works of Art, qui a sélectionné le projet de Simon Roberts : «L'implication du public dans le projet met en valeur le processus démocratique que Simon a entrepris de documenter ${ }^{30}$." D'après Simon Roberts, cet aspect de son projet a d'ailleurs été déterminant pour convaincre le comité qui l'a choisi comme artiste officiel des élections. Le photographe considère qu'en associant le public à l'œuvre que lui a commandée le Committee on Works of Art, il fait une «démocratie de la commande » (« a democracy of the commission »). À tous points de vue, le procédé participatif choisi pour The Election Project est bien en phase avec la recherche d'un renouveau de l'engagement des citoyens dans la vie démocratique qu'ont appelé de leurs vœux acteurs et observateurs de la vie politique en GrandeBretagne.

On peut aussi considérer que le dispositif déployé dans The Election Project relève d'une forme de « documentaire collaboratif ». Le concept est étudié notamment par Mandy Rose ${ }^{31}$, qui s'interroge sur le potentiel politique et social des formes culturelles collaboratives. Elle analyse les processus à l'œuvre dans le documentaire collaboratif comme une «DIY citizenship ", une " citoyenneté autoproduite ». En effet, les participants sont rassemblés, tout au moins virtuellement, par un objectif commun. Ils partagent leurs expériences et peuvent les comparer. C'est la nature même du documentaire que de générer un tel dialogue.

La galerie publique de The Election Project remplit donc une fonction d'échanges et aussi de mémoire collective, puisqu'elle devient une sorte d'archive visuelle des élections générales de 2010. Elle offre un lieu public de débat et de mémoire, invitant les participants à se joindre à une forme d'historiographie directe. Par comparaison avec la démarche de Mass Observation, qui sollicitait les observateurs de manière relativement isolée, The Election Project amène les participants à se confronter aux représentations produites par d'autres dès lors qu'ils alimentent l'archive commune, et éventuellement, à leur répondre par d'autres images. Il y a donc une forme de conversation entre participants, où les images remplacent les mots, comme c'est de plus en plus le cas aujourd'hui avec des applications telles que Snapchat.

45 Ce genre de projet documentaire participatif a donc un fort potentiel politique. Il stimule la citoyenneté, entendue non seulement comme un ensemble de droits et de devoirs du 
citoyen, mais aussi comme un investissement dans la vie sociale et politique. Un tel usage et une telle conception de la photographie ont été baptisés "nouveau documentalisme » par Jorge Ribalta. D'après lui, il faut remettre en avant la fonction sociale et politique de la photographie documentaire. Elle crée « une sphère publique déterritorialisée qui offre une condition générale de citoyen, partagée par tous sans distinction ${ }^{32}$. » De fait, The Election Project a mis en relation virtuelle une citoyenne de Salford, un citoyen de Hinchley Wood et des centaines d'autres contributeurs avec un photographe de Brighton et son public.

\section{Conclusion}

L'étude de plusieurs projets photographiques a permis de dégager les modes d'engagement de la photographie aujourd'hui : la figure du photographe engagé a fait place à la notion d'engagement participatif tandis que les thématiques se sont déplacées de la sphère sociale vers les questions d'identités individuelles et collectives, comme celle de l'intégration des réfugiés et immigrés en Grande-Bretagne, et celle de la citoyenneté et de la démocratie. Ces développements sont dus aux nouveaux outils de production numérique et de diffusion par les réseaux sociaux qui favorisent une désintermédiation et une démocratisation de la culture visuelle, même si la plupart des projets décrits s'appuient aussi sur l'implication de photographes professionnels. La photographie engagée a ainsi conquis d'autres terrains que ceux du photojournalisme et du simple documentaire après y avoir été remise en question. Il faut donc voir dans les deux dernières décennies un renouveau et une extension du domaine de la photographie engagée, mais surtout la production de nouveaux objets sociaux-culturels échappant aux catégories d'analyse de la politique ou de l'histoire de l'art et peut-être, de nouvelles sphères publiques de politisation.

Karine Chambefort-Kay (Université Paris Est Créteil, IMAGER/CIMMA) est l'auteur d'une thèse en civilisation britannique intitulée « Écritures photographiques des identités collectives : classe, ethnicité, nation dans la photographie britannique, 1990-2010 ». Ses recherches portent sur la culture visuelle contemporaine en Grande-Bretagne, et les usages sociaux des images en lien avec les questions d'identité, de mémoire et de mobilisation collective.

\section{BIBLIOGRAPHIE}

BERTRAND Mathilde, " "A Tool for Social Change" : Community Photography at Belfast Exposed ", Revue LISA/LISA e-journal, numéro thématique « Media, culture, histoire, Images et media », 2015. BOURRIAUD Nicolas, Esthétique relationnelle, Paris, Les Presse du Réel, 1998.

CHAMBEFORT Karine, «Écritures photographique des identités collectives : classe, ethnicité, nation dans la photographie en Grande-Bretagne, 1990-2010 ", thèse sous la direction de Didier Lassalle, soutenue en novembre 2014, à l'Université Paris Est Créteil. 
CHERoux Clément, "Mythologie du photographe de guerre", in GERVEREAu Laurent et al. (dir.), Voir/Ne pas voir la guerre. Histoire des représentations photographiques de la guerre, Paris, BDIC / Somogy, 2001, p. 306-311.

JONES GRIFFITH Phillip, Vietnam Inc., New York, Collier, 1971.

MAYNE Roger, Photographs, Londres, Jonathan Cape, 2001.

MCCULLIN Don, Don McCullin in Africa, London, Jonathan Cape, 2005.

PANZER Mary (dir.), Things as They Are. Photojournalism in Context Since 1955, New York, Aperture Foundation / World Press Photo, 2005.

Photovoice /DOST, New Londoners : Reflections on Home, Londres, Trolley, 2009.

RIBALTA Jorge, « Towards a New Documentalism », Variant n 43, Printemps 2012, http:// www.variant.org.uk/43texts/JorgeRibalta43.html.

RITCHIN Fred, «La proximité du témoignage. Les engagements du photojournaliste », in FRIZOT Michel (dir.), Nouvelle histoire de la photographie, Paris, Bordas, 1994, p. 590-609.

ROSE Mandy, «Documentary, Open Space, Citizenship - "Making Publics"? », i-Docs Symposium, Digital Cultures Research Centre, University of the West of England, mars 2012.

schuman Aron et TOMLInson Alys, Photodebut : A Case Study, Londres, The Photographer's Office, 2009.

SEKULA Allan, « Dismantling Modernism, Reinventing Documentary (Notes on the Politics of Representation) », The Massachusetts Review, volume 19, n 4, « Photography », hiver 1978.

SONTAG Susan, Sur la photographie, Paris, Christian Bourgeois/ Titres, 1993.

\section{NOTES}

1. Voir CHÉROUX Clément, "Mythologie du photographe de guerre", in GERVEREAU Laurent et al. (dir.), Voir/Ne pas voir la guerre. Histoire des représentations photographiques de la guerre, Paris, BDIC / Somogy, 2001, p. 306-311.

2. «Ces dernières décennies, la photographie "engagée" a au moins fait autant pour émousser la conscience que pour l'aiguiser." SONTAG Susan, Sur la photographie, Paris, Christian Bourgeois/ Titres, 1993, p. 39.

3. RITCHIN Fred, «La proximité du témoignage. Les engagements du photojournaliste ", in FRIzOT Michel (dir.), Nouvelle histoire de la photographie, Paris, Bordas, 1994, p. 590-609.

4. JONES GRIFFITH Phillip, Vietnam Inc., New York, Collier, 1971.

5. MCCULLIN Don, Don McCullin in Africa, London, Jonathan Cape, 2005.

6. PANZER Mary (dir.), Things as They Are. Photojournalism in Context Since 1955, New York, Aperture Foundation / World Press Photo, 2005.

7. « in simple terms to all observers, so that their environment may be understood, and thus constantly transformed. Whatever the political methods called upon to effect the transformation, the knowledge of has to be transformed is indispensable. " HARRISSON Tom, JENNINGS Humphrey et MADGE Charles, fondateurs de Mass Observation, lettre aux journaux The New Statesman et The Nation, 30 janvier, 1937.

8. MAYNE Roger, Photographs, Londres, Jonathan Cape, 2001. 
9. "shielded by a bogus ideology of neutrality», SEKULA Allan, "Dismantling Modernism, Reinventing Documentary (Notes on the Politics of Representation)», The Massachusetts Review, volume 19, $\mathrm{n}^{\circ} 4$, « Photography », hiver 1978, p. 860.

10. Voir par exemple BERTRAND Mathilde, " "A Tool for Social Change" : Community Photography at Belfast Exposed », Revue LISA/LISA e-journal [En ligne], Media, culture, histoire, Images et média, mis en ligne le 21 juillet 2015.

11. CHAMBEFORT Karine, «Écritures photographique des identités collectives : classe, ethnicité, nation dans la photographie en Grande-Bretagne, 1990-2010 ", thèse sous la direction de Didier Lassalle, soutenue en novembre 2014, Université Paris Est Créteil.

12. Brighton Photo Biennal, 4 octobre-2 novembre 2014.

13. Martin Parr, Daniel Meadows, Sirkka Liisa Konttinen, Nick Hedges, Fay Goodwin, Paul Hill, Ron Mc Cormick, Gerry Badger.

14. ABC, Burn My Eye, Ruido Photo, Sputnik Photos, Uncertain States.

15. " to outline the impact of this way of working, nationally and internationally. Considered here is the dynamic, democratic process of navigating and negotiating a cohesive voice through the notion of a collective. » Programme Brighton Photo Biennal 2014, p. 10.

16. «Photoworks, an organization dedicated to enabling participation in photography, the most democratic medium in contemporary visual culture.", page de presentation de l'agence Photoworks, https://photoworks.org.uk/About, consultée le 2 mars 2017.

17. schuman Aaron et tomLinson Alys, Photodebut: A Case Study, Londres, The Photographer's Office, 2009.

18. «We are passionate about documentary photography and we are committed to photographing Scotland. [...] Now, in a sense, Caledonia is calling. [...] Scotland today stands at a decisive moment in its history. Events over the next few years will shape how we relate to neighbours and to the wider world. Document Scotland believe that photography can and should play a central part in documenting this epoch. Our aspiration is to make work and engage in a discourse which will form a vital component of the history and conversation of our nation tomorrow. We hope to leave a visual document, a testimony to the extraordinary times we are living in.» Page «About» $\mathrm{du}$ site $\mathrm{du}$ collectif Document Scotland: http:// www.documentscotland.com/scottish-documentary-photography-about/, consultée le 2 mars 2017.

19. BOURRIAUd Nicolas, Esthétique relationnelle, Paris, Les Presse du Réel, 1998, p. 117.

20. Les photographes professionnels ayant participé au projet sont Adam Broomberg, Oliver Chanarin, Gayle Chong Kwan, Marysa Dowling, Suki Dhanda, Jillian Edelstein, Liane Harris, Crispin Hughes, Anna Kari, Anthony Lam, Jenny Matthews, Jo Metson Scott, Sarah Moon et Ilona Suschitsky, et Othello de Souza Hartley.

21. Photovoice / DOST, New Londoners : Reflections on Home, Londres, Trolley, 2009.

22. " Taking pictures is a way of establishing that a new life does have a form, for confronting the looming shadows and celebrating what's beautiful. These pictures are memories - and when you have memories of a place, you're beginning to put down roots. » Hari KUNZRU, «Introductions », New Londoners, Op. Cit., non paginé.

23. "Maybe I'll be happy here. I have not chosen to be here, and have no choice if they want to send me back. I should be happy but I'm not. I'm not able to start my life yet. Not until I get my visa. » Chalak Abdulrahman, New Londoners, non paginé.

24. "Our ambition is to help re-frame the debate about asylum away from fear, hostility and difference and towards commonality and recognition." Liz ORTON, "Photography and Integration: a Case Study of a Photovoice Project", in Stella Barnes, Participatory Arts with Young Refugees, Six Essays Collected and Published by Oval House Theatre, Londres, Arts in Education, Oval House Theatre, 2009, p. 2. 
25. «My first impressions of London were strange. I was in East Ham, and it was not like the London I had seen on the television. The experience of seeing different customs and lifestyles was new. After a while I got to know about these cultures and I came to respect them. Once you get over the strangeness of the difference you understand how many things are the same.» Bajram Spahia, dans New Londoners, Op. Cit., non paginé.

26. «the photographs are also about transition - from one place to another, from dependence to independence, and from one way of life to another. » Tiffany FAIREY et Liz ORTON, « Foreword", New Londoners, Op. Cit., non paginé.

27. «Our campaign seeks to challenge the negative rhetoric against immigrants, celebrate them and provide them with a platform to share their story. "

28. Site www.iamanimmigrant.net, consulté le 2 mars 2017.

29. «Utilizing photographs taken by the public, uploaded from digital cameras or transmitted via mobile phones, will create an alternative photographic vision alongside my own captured via the more traditional means of [his] plate camera, and will add a collaborative and democratic dimension to the overall work. They will also help to provide an antidote to the more stagemanaged photographs increasingly seen from the campaign trail. » Simon Roberts, cité dans «Official election artist wants voters to get involved », British Parliament, 12 avril 2010, http:// www.parliament.uk/business/news/2010/04/official-election-artist-wants-voters-to-getinvolved/, consulté le 15 mars 2013.

30. "Getting the public involved in the project also emphasises the democratic process Simon is documenting. " Hugo SWIRE, cité dans "Simon Roberts announced as 2010's official election artist », National Media Museum, $1^{\text {er }}$ avril 2010, http://www.nationalmediamuseum.org.uk/ AboutUs/PressOffice/2010/April/SimonRobertsElectionArtist.aspx, consulté le 15 mars 2013.

31. ROSE Mandy, "Collabdocs ", http://collabdocs.wordpress.com/, consulté le 15 mars 2013, et du même auteur: "Documentary, Open Space, Citizenship - "Making Publics"? », i-Docs Symposium, Digital Cultures Research Centre, University of the West of England, mars 2012.

32. «A de-territorialised public sphere that offers a general and equally shared condition of citizenship ", RIBALTA Jorge, "Towards a New Documentalism ", Variant n 43, Printemps 2012, [En ligne] http://www.variant.org.uk/43texts/JorgeRibalta43.html, consulté le 15 mars 2013.

\section{RÉSUMÉS}

La figure héroïque du photographe engagé semble avoir disparu depuis les années 80, avec comme tournant symbolique l'entrée de l'artiste Martin Parr à l'agence Magnum en 1991. Cela ne signifie pas pour autant que le principe de l'engagement ait été évacué des pratiques photographiques contemporaines en Grande-Bretagne. Si une multitude de collectifs de photographes se sont créés à partir des années 2000, évocateurs des pratiques militantes antérieures, ceux-là ne sont pas les acteurs privilégiés du renouveau de l'engagement par la photographie, que nous situons plutôt dans des pratiques participatives et des projets déployés à la marge des champs artistiques et journalistiques. À travers trois études de cas successives - le projet New Londoners, de l'association Photovoice, la campagne I Am an Immigrant de Mouvement Against Xenophobia et The Election Project de Simon Roberts. - nous dégageons les modalités d'engagements par la photographie qui mettent à profit les outils de production numérique et de diffusion par les réseaux sociaux. De la participation à l'émancipation individuelle, de 
l'identification à la mobilisation collective et enfin du photojournalisme citoyen à la constitution de nouvelles sphères publiques d'interactions politiques, nous voyons dans de tels projets une réactivation du potentiel unique d'engagement de la photographie quelque peu perdu de vue dans les deux décennies précédentes.

Since the late 1980s, the heroic figure of the committed photojournalist has waned from the world of photography, with the nomination of Martin Parr as a member of Magnum Photos in 1991 as a symbolic turning point. Yet this does not mean that a sense of concern and commitment cannot be found in contemporary photographic practices. Many photographers' collectives have been created since the 2000s that may remind us of radical groups from previous decades, though few of them participate in the renewal of committed photographic practices. Instead, it is argued, commitment lies in participatory projects developed outside the fields of art and photojournalism. Three cases are studied in this article - New Londoners, by Photovoice, the "I Am An Immigrant" campaign from the Movement Against Xenophobia and Simon Roberts's Election Project - in an attempt to pin down new modes of commitment through photographs, especially involving digital media and social networks. From participation to individual empowerment, from collective identification to mobilization, from citizens' photojournalism to the creation of public spheres for political interaction, photography's unique potential for political commitment, having arguably been lost in the past two decades, has been reactivated.

\section{INDEX}

Mots-clés : photographie, collectifs de photographes, Photovoice, I Am an Immigrant campaign, Simon Roberts, militantisme

Keywords : photography, photography collectives, Photovoice, I Am An Immigrant campaign, Simon Roberts, activism

\section{AUTEUR}

\section{KARINE CHAMBEFORT-KAY}

IMAGER/Cimma, Université Paris Est Créteil 